\title{
Online teaching of basic surgical skills to medical students during the COVID-19 pandemic: a case-control study
}

\author{
Michael $\mathrm{Co}^{1} \cdot$ Patrick Ho-Yu Chung ${ }^{1} \cdot$ Kent-Man $\mathrm{Chu}^{1}$
}

Received: 29 October 2020 / Accepted: 3 December 2020 / Published online: 25 January 2021

(c) Springer Nature Singapore Pte Ltd. 2021

\begin{abstract}
Purpose Medical education has been disrupted by the COVID-19 pandemic in many countries, with face-to-face lectures replaced by pre-recorded videos. However, surgical skills training cannot be replaced easily by videos, as a high level of tutor-student interaction is required. Thus, we developed a new web-based surgical skill learning session (WSSL). This case-control study evaluates the surgical skills competency of medical students taught by the WSSL.

Methods This case-control study compares WSSL with face-to-face tutorials. Students were assigned randomly to one of two groups according to the teaching method. Independent blinded assessment was performed by a standardized marking scheme, modified from the Objective Structured Assessment of Technical Skills (OSATS) global rating scale.

Results We recruited 62 final-year medical students into the study, with 33 randomized to the face-to-face teaching group (control group), and 29 to the WSSL group(case group) according to their student number. The baseline demographic characteristics of the two groups were comparable. The mean score at the clinical competency assessment of the control group was 4.8/5 (range 4-5) and that of the case group was $4.7 / 5$ (range 4-5) $(p=1)$. There were no difficulties with program or hardware installation reported by the WSSL students.

Conclusions Surgical skills performance was comparable between students who were taught by the WSSL and those taught by conventional face-to-face tutorials.
\end{abstract}

Keywords Telecommunication $\cdot$ Surgical skills $\cdot$ Undergraduate

\section{Introduction}

An outbreak of coronavirus disease 2019 (COVID-19) was identified in Wuhan, China in December 2019 [1], subsequent to which the virus spread quickly, resulting in a pandemic across 210 countries. By October 8, 2020, more than 32 million cases had been recorded worldwide. Most countries implemented social distancing measures to contain the infection, including the closure of learning institutions from primary schools to universities [2]. Medical education in Hong Kong has been impacted severely as medical students can no longer participate in any clinical activity and must attend classes online from home, to comply with the social

Kent-Man Chu

chukm@hku.hk

1 Center for Education and Training, Department of Surgery, The University of Hong Kong, Queen Mary Hospital, Pok Fu Lam, Hong Kong distancing measures implemented during the COVID-19 outbreak.

The medical curriculum in Hong Kong comprises 3 years of pre-clinical teaching and another 3 years of clinical teaching, followed by a 1-year internship (house officer). Basic surgical skills, including making simple incisions, suturing, and surgical knot-tying (instrumental and manual) are normally taught by a general surgeon (First author MC, or his delegate) face-to-face in an interactive tutorial to students in their final year of medical school. The students are divided into seven groups of approximately 30 . This face-to-face interactive basic surgical skills tutorial has been in effect for more than 20 years in our institution. Each medical student is given a piece of synthetic skin and a "suture set", comprised of a scalpel, scissors, needle holder, forceps, and suture materials. They are allowed to practice suturing in front of the tutor (MC). The interactive tutorial normally lasts for $120 \mathrm{~min}$ (30 $\mathrm{min}$ for the demonstration and $90 \mathrm{~min}$ for hands-on practice). 
Lectures are now delivered online via the videoconferencing platform "Zoom" (Zoom Video Communications, Inc. San Jose, CA, USA), operating theatre and endoscopy center attachments have been replaced by online video demonstration, and clinical case studies have been replaced by online tutorials. The basic surgical skills interactive tutorial has also been replaced by online teaching. Students are asked to collect their surgical instruments from the faculty office and attend the surgical skills teaching online from home. Web-based surgical skills learning (WSSL) was developed and implemented in the final year surgical curriculum in March 2020 [3].

\section{Methods}

This is a prospective case control study on student's performance, comparing the competency of those who were taught by WSSL (case group) with those who received conventional face-to-face teaching (control group).

\section{Subjects}

The study was conducted in June 2020, when face-to-face teaching was allowed by the Government as the number of COVID-19 cases had decreased. Two consecutive rotations of final year medical students were recruited for the study and all participants gave their informed consent. Each rotation of students was divided into two groups according to the student number. The first group received $120 \mathrm{~min}$ of WSSL and the second group received $120 \mathrm{~min}$ of face-to-face teaching by the same tutor. Both groups were given the same teaching content with a standardized syllabus. All students were assessed for their competency in making a linear incision, suturing, and tying a correct surgical knot (square knot) 3 weeks after the teaching at the end of the surgical rotation.

\section{Control group (conventional face to face teaching)}

Each medical student was given a piece of synthetic skin and a suture set (scissors, needle holder, forceps and suture materials). Surgical skills such as making a skin incision, suturing, and knot tying were taught face-to-face (Fig. 1). Students were then allowed to practice suturing in front of the tutor. The duration of the interactive tutorial was $120 \mathrm{~min}$ (30 min for demonstration and $90 \mathrm{~min}$ for handson practice).

\section{Case group (WSSL)}

As with the control group, surgical instruments and synthetic skin were distributed to the students prior to the session for real-time practice of incision, suturing, and knot-tying. The

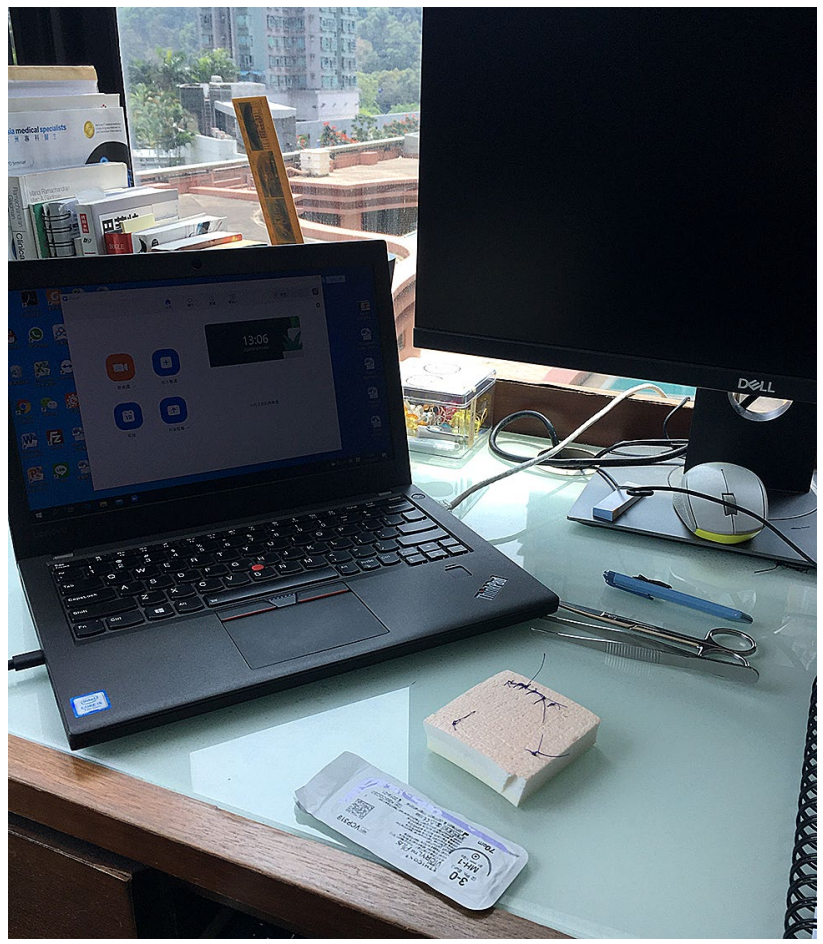

Fig. 1 Setup for the WSSL at home. Photo credit to medical student Mr. Kelvin Zheng

WSSL session also lasted for $120 \mathrm{~min}$ (30 min for the tutor demonstration and $90 \mathrm{~min}$ for hands-on practice by the student). Unlike conventional face-to-face teaching, students participated in the WSSL via Zoom at home (Fig. 2).

Two cameras were used to broadcast the session live from the tutor's location. Camera one was used for close-up capturing of the tutor's hands to demonstrate the surgical skills and techniques clearly. Camera two was the built-in camera on the computer, which was used for the usual video-conference focusing on the tutor's face. Each camera was linked to individual Zoom ${ }^{\mathrm{TM}}$ accounts so that the students (the viewers) were able to see the tutor's face and hands simultaneously on the same screen. Camera one was placed behind the tutor to prevent mirror imaging of the hand movements (Fig. 3) [3]. Students were then asked to demonstrate their surgical skills by setting up a camera at home, which allowed real-time feedback from the tutor, to mimic the tutor-student interaction during a face-to-face session [3].

\section{Primary endpoints}

An independent assessor was blinded to which teaching method each student had received and standardized criteria were used to assess surgical skills competency. The marking scheme was modified from the Objective Structured Assessment of Technical Skills (OSATS) global rating scale described previously [4]. The OSATS global rating 
Fig. 2 Setup for conventional face-to-face surgical skills teaching

Fig. 3 Setup for the web-based surgical skills learning session (WSSL)
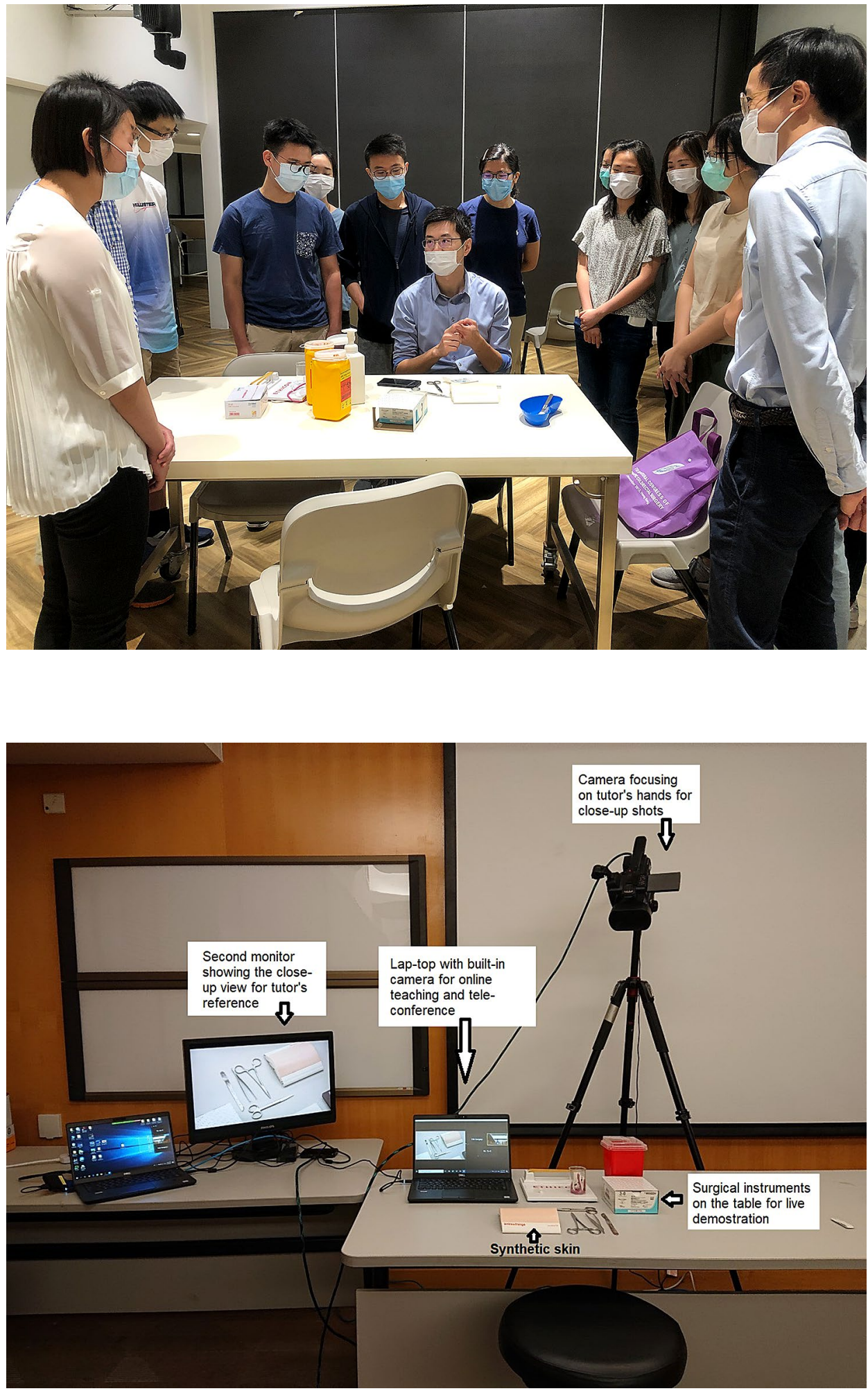

scale was designed and validated to evaluate the skills of surgical trainees in the operating room [4]. The modified OSATS was used to tailor the needs for assessing undergraduate students (Fig. 4).

\section{Statistical analysis}

Demographic characteristics and scores of the clinical competency assessment of students between the two groups were 
Fig. 4 Marking scheme for blinded assessment of the students' performance. Modified OSATS global rating scale

\section{Student Name}

Please put label here

\section{Web-based Surgical Skills Learning (WSSL)}

Clinical Competency Assessment

\section{Instruction:}

Please perform linear skin incision followed by skin suturing.

Marking scheme (Please circle where appropriate):

\begin{tabular}{|l|l|l|l|}
\hline Item & $\begin{array}{l}\text { Properly performed } \\
1 \text { point }\end{array}$ & $\begin{array}{l}\text { Fairly performed } \\
0.5 \text { point }\end{array}$ & $\begin{array}{l}\text { Unsatisfactory } \\
0 \text { point }\end{array}$ \\
\hline Respect of tissue & & & \\
\hline Time and motion & & & \\
\hline Instrument handling & & & \\
\hline Knowledge of instruments & & & \\
\hline Flow of operation & & & \\
\hline
\end{tabular}

Comments (if any):

Total score:

Modified OSATS

Adopted in May 2020

compared by the Fishers exact test, Chi-square test, or Student's $T$ test where appropriate.

\section{Results}

A total of 62 medical students were recruited into the study (median age, 23 years old; range 22-24 years old). There were 28 female and 34 male students, all of whom were in their final year and do not have other university degree or professional qualifications. Sixty students were right-handed and two were left-handed. Two face-to-face teaching sessions and two WSSL sessions were arranged for the $33 \mathrm{stu}-$ dents in the control group and the 29 students in the case group, respectively.

Table 1 summarizes the baseline demographic data of the two groups. All students were able to perform a proper surgical knot using an instrumental tie. The mean score at the clinical competency assessment (modified OSATS) of the control group was 4.8/5 (range 4-5) and that of the case
Table 1 Baseline demographic data of the case and control groups

\begin{tabular}{llll}
\hline Factor & Case group (WSSL), $N=29$ & $\begin{array}{l}\text { Control group (face-to- } \\
\text { face learning), } N=33\end{array}$ & $p$ value \\
\hline Gender & Male $=17(58.6 \%)$ & Male $=17(51.5 \%)$ & 0.6170 \\
& Female $=12(41.4 \%)$ & Female $=16(48.5 \%)$ & \\
Postgraduate degree & No=29 $(100 \%)$ & No=33 $(100 \%)$ & 1 \\
& Yes=0 $(0 \%)$ & Yes=0 $(0 \%)$ & \\
Handedness & Left $=1(3.4 \%)$ & Left $=1(3.3 \%)$ & 1 \\
& Right $=28(96.6 \%)$ & Right $=32(96.7 \%)$ & \\
& Ambidextrous $=0$ & Ambidextrous $=0$ & \\
History of failure in surgical examina- & No=29 $(100 \%)$ & No=33(100\%) & 1 \\
tions (paper or clinical examinations) & Yes=0 $(0 \%)$ & Yes=0 $(0 \%)$ & \\
\hline
\end{tabular}


group was 4.7/5 (range 4-5) ( $p=1$ ). Subgroup analyses were done of the students' performance in each individual assessment item in the modified OSATS; namely, in respect of tissue, time and motion, instrument handling, knowledge of instruments, and flow of operation. There was no significant difference between the study and control groups. The WSSL students reported no difficulties with the program or hardware installation.

\section{Discussion}

COVID-19 is a highly contagious disease and in the absence of effective disease control and treatment, the only strategy against COVID-19 at the time of study is to prevent the mixing of susceptible and infected people through the early identification of cases and reduction of the risk of contact [5]. Schools and universities in many affected countries were closed to slow down the spread of the disease and learning sessions that had been normally delivered on campus were replaced by online teaching [2,5].

Medical education has adopted the same measures; however, unlike for primary or secondary education, delayed graduation from medical schools can have disastrous consequences during the COVID-19 pandemic [6-8]. While lectures can be delivered online easily, surgical skills training, which requires a high level of teacher-student interaction, may be challenging to demonstrate via pre-recorded videos [3]. Thus, our institution has developed a new online webbased interactive surgical training session to overcome the problem. Lectures that were normally taught face-to-face have been delivered online during the outbreak, either by a live lecture or by pre-recorded videos.

Here we described a novel surgical skills training strategy delivered through a commercially available online platform. Students were instructed not to dispose of sharps in their domestic garbage, but were required to collect all used suture needles and scalpel blades and dispose of them in the sharps boxes provided by the University. All students were able to demonstrate correct handling of surgical instruments and knot-tying. This web-based surgical skills learning has overcome two important obstacles during the COVID-19 pandemic: the lack of face-to-face interaction as well as realtime feedback during the learning. The alternative of providing a pre-recorded video demonstrating the same skills would not have overcome the two obstacles. WSSL was delivered with real-time skills demonstration by two cameras, one focusing on tutor's face and the second one zooming in on the tutor's hands. The camera angle and power of magnification of tutor's hands could be adjusted at any time. Students were allowed to make comments or ask questions throughout the WSSL session and were also asked to demonstrate surgical tasks via the same online platform. Another important advantage of WSSL is its ability to demonstrate the skills so clearly. Unlike in face-to-face tutorials, where some students are sitting at the back of the room and may have a limited view, WSSL allows for the demonstration of surgical skills to all students equally.

WSSL is affordable, it does not require a highly specialized software program or professional equipment apart from the usual video conferencing platform and there is no additional cost for equipment setup. However, there are some limitations with this teaching method. First, not all medical students, especially those living in economically lessprivileged areas, may have internet access and a personal computer. Second, the time difference can be a potential problem if the medical students are attending classes from different time zones, which is particularly relevant for universities with a large number of overseas students. Nevertheless, these limitations can be overcome by measures such as allowing students to attend classes using computers in the library and scheduling multiple classes to accommodate students from different time zones. Ultimately, we believe that online teaching will enhance medical education, including surgical skills training for students and resident trainees (9), even after the COVID-19 pandemic.

\section{Conclusion}

Surgical skills performance was comparable between students who were taught by WSSL and those who were taught by a conventional face-to-face tutorial.

Author contributions MC: conceptualization, data acquisition, data analysis and interpretation of data, drafting of the manuscript. PC: conceptualization, data acquisition, final editing. K-MC: conceptualization, supervision, final editing.

Funding This study was not sponsored by any external funding.

\section{Compliance with ethical standards}

Conflict of interest We have no conflicts of interest to declare.

\section{References}

1. Huang C, Wang Y, Li X, Ren L, Zhao J, Hu Y, et al. Clinical features of patients infected with 2019 novel coronavirus in Wuhan, China. Lancet. 2020;395:497-506.

2. United Nations Educational, Scientific and Cultural Organization. COVID-19 educational disruption and response. 2020. https:// en.unesco.org/themes/education-emergencies/coronavirus-schoo 1-closures. Accessed 12h Apr 2020

3. Co M, Chu KM. Distant Surgical Teaching during COVID-19-a pilot study on final year medical students. Surg Pract (Published 22 Jun 2020). 
4. Niitsu H, Hirabayashi N, Yoshimitsu M, Mimura T, Taomoto J, Sugiyama Y, et al. Using the Objective Structured Assessment of Technical Skills (OSATS) global rating scale to evaluate the skills of surgical trainees in the operating room. Surg Today. 2013;43(3):271-5.

5. Lewnard JA, Lo NC. Scientific and ethical basis for socialdistancing interventions against COVID-19. Lancet Infect Dis. 2020;20(6):631-3.

6. Cambridge University: all lectures to be online-only until summer of 2021. BBC (Family and Education) 19 May 2020. https://www. bbc.com/news/education-52732814. Accessed 1 Jun 2020

7. DeWitt DE. Fighting COVID-19: enabling graduating students to start internship early at their own medical school. Ann Intern Med. 2020; M20-1262 [published online ahead of print, 2020 Apr 7].
8. Chick RC, Clifton GT, Peace KM, Propper BW, Hale DF, Alseidi AA, et al. Using technology to maintain the education of residents during the COVID-19 pandemic. J Surg Edu. 2020;77(4):729-32.

9. Gregg SC, Duffy EAJ, Longo WE. Design, management, and critical evaluation of a surgical basic/clinical science curriculum: the role of an educational chief resident. J Surg Educ. 2008;65:36-42.

Publisher's Note Springer Nature remains neutral with regard to jurisdictional claims in published maps and institutional affiliations. 